\title{
A model based system for control of live feed level for larval fish
}

\author{
D. SLAGSTAD, $\dagger$ Y. OLSEN $\ddagger$ and S. TILSETH $\ddagger$
}

Keywords: Biocybernetics, aquaculture, fish larvae, plankton, modeling, sensitivity analysis.

A mathematical model of the production process of live feed to fish larvae is presented. The model is used as a design tool to construct a control system which ensures stable and controllable conditions for live feed organisms (rotifers). The simulation results demonstrate the effect of possible changes in the biological parameters and different operational routines.

\section{Introduction}

Modeling and simulation of dynamic system are widely used in study of complex industrial and biological processes. There are many reasons why modeling and simulation should be performed. The modeling process itself forces one to evaluate which parts of the process are important according to the objective of the model. Areas where quantitative knowledge are missing will soon be uncovered. Simulations on a computer will give knowledge about the dynamics of the system i.e. time response of the variables after an initial condition or a perturbation of one or several variables.

In this paper the modeling and simulation technique will be used for studying the production process of rotifers which is used as live feed for first feeding of fish larvae. The early feeding of fish larvae requires live feed of suitable quality and concentration. The size range and the concentration of live feed must stay near some optimum value. This value changes as the fish larvae grows. Optimal conditions for larval growth are only partly known, but the objective with most of the systems used for production of fish larvae is to keep the conditions as near optimum as possible.

The need for live feed increases as the fish larvae increase in size and the production of live feed should always be such that it can keep track with the needs and a minimum of over-production.

\section{The model equations}

Rotifers are assumed to be produced in a semi-continuous culture where a certain fraction of the water volume is harvested at specified time intervals. Rotifers

Received 15 January 1987.

This paper was presented at the IFAC Symposium on Automation and Dataprocessing in Aquaculture, Trondheim, Norway, 19-20 August 1986.

This paper is reprinted with the permission of IFAC.

$\dagger$ Division of Automatic Control, The Foundation for Scientific and Industrial Research at the Norwegian Institute of Technology (SINTEF), N-7034 Trondheim-NTH, Norway.

$\ddagger$ Division of Applied Chemistry, The Foundation for Scientific and Industrial Research at the Norwegian Institute of Technology (SINTEF), N-7034 Trondheim-NTH, Norway. 
reproduce by nonsexual egg production. When proper conditions are given, the eggs grow to adults and start reproduction within 36 hours. In a batch culture we thus find a size distribution of rotifers. In steady state conditions, i.e. when the harvest and new production are equal, this distribution is fixed, independent of time. Under dynamic conditions the size distribution is continuously changing and can have an effect on the production in the next 24 to 48 hours. Size distribution is therefore a property that should be included in the model.

The dynamics of the size distribution can be described by the following partial differential equation, see Slagstad (1981) for further information.

$$
\frac{\partial N_{r}(t, w)}{\partial t}+g_{r} \frac{\partial N_{r}(t, w)}{\partial w}=0
$$

where $N_{r}(t, w)$ is the number of rotifers per litre with weight $w$ at time $t . g_{r}$ is the growth rate for an animal of weight $w$. The growth rate will also depend on temperature and the concentration of proper food (flagellates) for the rotifers.

When the rotifers become adults, we assume that growth ceases and egg production starts immediately at a fixed rate (see Table 1). The adult rotifers are assumed to have an energy content of $8 \times 10^{-4} \mathrm{cal}$ equivalent to $0.5 \mu \mathrm{g}$ dry wt. (Theilacker and McMaster, 1971) and the eggs 1.3 $\times 10^{-4} \mathrm{cal}$ (unpublished data).

\begin{tabular}{llll}
\hline Parameter & Unit & Value & \\
\hline$N_{\text {egg }}$ & $\mathrm{d}^{-1}$ & $2 \cdot 0$ & Rate of egg production per rotifer \\
$g_{r}$ & $\mathrm{~d}^{-1}$ & $0 \cdot 8$ & $\begin{array}{l}\text { Growth rate } \\
A_{s w}\end{array}$ \\
$T_{\text {d }}$ & $16 \cdot 0$ & $\begin{array}{l}\text { Age when switching from rotifer to other food } \\
\text { particles start }\end{array}$ \\
$f_{\text {hmx }}$ & d & $5 \cdot 0$ & $\begin{array}{l}\text { Time constant for the switching process } \\
S_{p}\end{array}$ \\
\hline
\end{tabular}

Table 1. Tentative values of parameters used in the simulation runs

The steady state size distribution, i.e. obtained when a constant fraction of all size groups is harvested daily so that the number of animals stay constant, is shown in Fig. 1. This Fig. shows that most of the animals are adults. Since the animals have a short generation time, they spend only a small proportion of their life time in a non-adult stage.

Growth of fish is not modeled but a fixed relationship between larval age and food demand is assumed, see Table 2. The larval age is zero at the time of hatching.

\begin{tabular}{lllllll}
\hline $\begin{array}{l}\text { Larval age } \\
\text { [days] }\end{array}$ & 0 & 5 & 20 & 30 & 40 & 60 \\
\hline $\begin{array}{l}\text { Energy demand } \\
{\left[\text { cal day }^{-1} \text { ] }\right.}\end{array}$ & 0 & 0.11 & 0.19 & 0.32 & 0.52 & 1.42 \\
\hline
\end{tabular}

Table 2. Relationship between energy demand and larval age. (Data calculated from Erlich, 1974). 


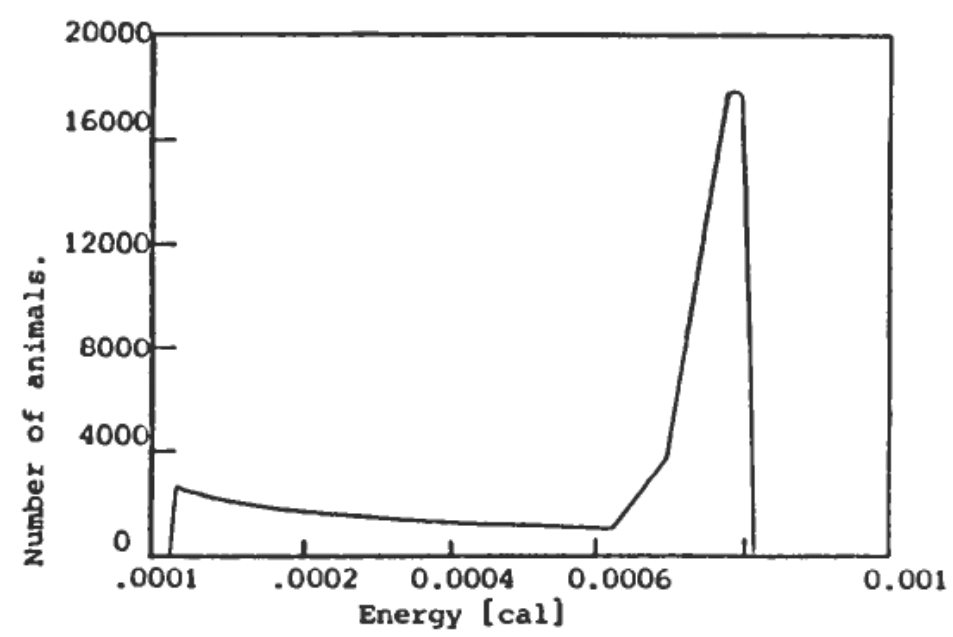

Figure 1. Size distribution of rotifers in a steady state situation.

At a certain age, $A_{s w}$, the larvae start switching from rotifers to an alternative food source. We simulate this switching by using the function:

$$
R_{f r}= \begin{cases}1.0 & a \leqslant A_{s w} \\ 1.0-\exp \left[-\left(A_{s w}-a\right) / T_{\text {rot }}\right] & a>A_{s w}\end{cases}
$$

where $R_{f r}$ is the relative fraction of the larval energy demand which is taken from rotifers diet, $a$ is larval age, $A_{s w}$ is age when switching to a new diet and $T_{\text {rot }}$ is the time constant for the switching process. (A time constant equal 10 days means that $37 \%$ of the larval energy demand is covered by rotifers at day 10 from the switching day). The parameter used in the simulation runs are given in Table 1.

Using Table 1 and Eqn. (2) we can plot the demand for rotifers as a function of time (Fig. 2).

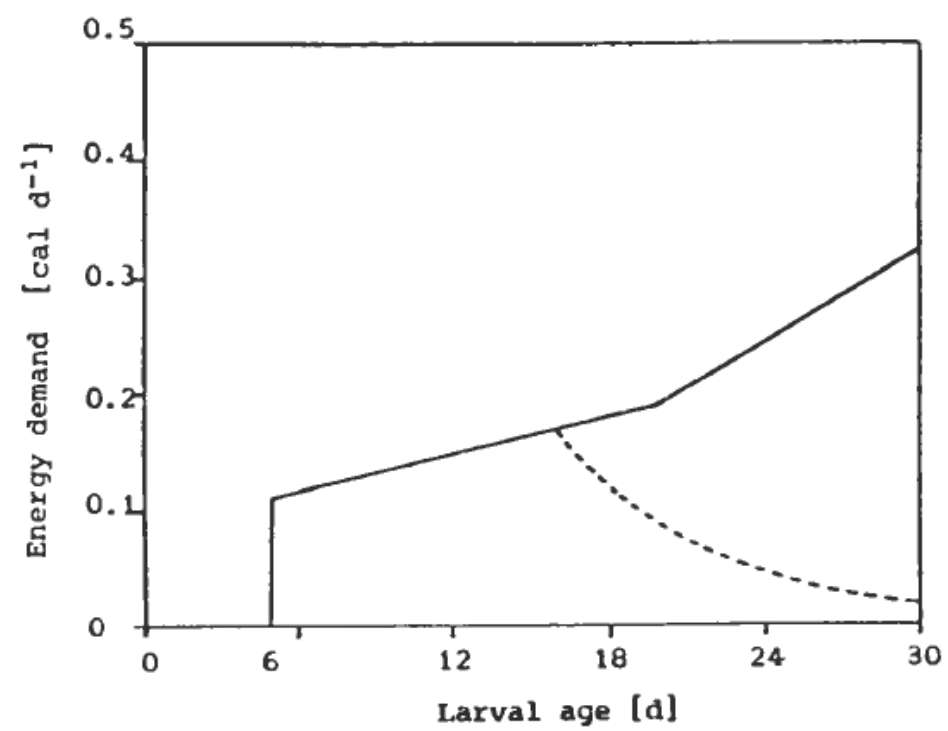

Figure 2. Energy demand of a single fish larvae (solid line) and energy taken from rotifers (broken line) as a function of time. 


\section{Control system}

We assume that the measurement that is available from the rotifers' vessel is the concentration i.e. number of rotifers per litre, and that there is no information about size distribution.

To control the rotifers' concentration there are several possibilities, (a) change growth rate by changing food supply or temperature, $(b)$ harvest a certain fraction of the total production volume in specified time intervals and $(c)$ harvest a certain size fraction of rotifers.

The control strategy will be as follows. It is assumed that there is a maximum concentration of rotifers above which the growth rate will be sub-optimal. When the rotifers' vessel is sampled (i.e. measurement of concentration and harvesting) start concentration and water volume is calculated in order to have the rotifers' concentration which is near maximum at the next time of sampling. The total amount of rotifers should be enough to satisfy the demand from the fish larvae and ensure a start concentration which is high enough to cover future needs.

The control algorithm for the discrete PID-controller is:

$$
\begin{gathered}
u_{k}=u_{k-1}+k_{p}\left(e_{k}-e_{k-1}+0.5 T_{\text {samp }}\left(e_{k}+e_{k-1}\right) / T_{i}\right) \\
e_{k}=\left(y_{\text {ref }}-y_{k}\right) / y_{\text {ref }}
\end{gathered}
$$

where $u$ is the controller output, $y$ is a measurement (in this case number of rotifers per litre), $k_{p}$ and $T_{i}$ are parameters that are given values according to the time constants of the system that is controlled and $y_{\text {ref }}$ is a reference concentration. The index $k$ indicates that the variable values are taken at present time whereas the index $k-1$ indicates the variable values at the previous sample.

\section{Results and discussion}

The simulation model is in an early stage of development and improvements can easily be included. Variable input of food (flagellates) to control growth of rotifers and measurement of actual rotifer concentration in the fish larval vessel are among such improvements. However, in spite of the shortcomings, this mathematical model has been a powerful tool for design of working routines during operation.

The model has been simulated on a VAX-750 computer using a combined discrete/continuous simulation language, ACSL, for solving the mathematical equations and presenting the simulation results. (Mitchell and Gauthier, 1981).

At the chosen conditions, the production process of rotifers is unstable which mean that it is not possible to control the rotifers' concentration without any measurements and a proper control algorithm. Any variation in the growth or reproduction parameters will cause an increase or decrease in the rotifers' concentration. As an example of how sensitive the rotifers production is to variation in the reproductive rate we have made the following experiment.

We assume that the time from hatching to maturity for the rotifers is 36 hours and the rate of egg production for an adult rotifer is equal to $2 \mathrm{~d}^{-1}$. This gives a doubling rate of the population equal to $1.02 \mathrm{~d}^{-1}$. To keep the concentration in a steady state it is necessary to harvest $50.8 \%$ of the production volume each day.

If the egg production rate is increased by $10 \%$, the doubling rate of the population will increase. Keeping the harvest fraction constant the concentration of rotifers will increase as shown in Fig. 3. 


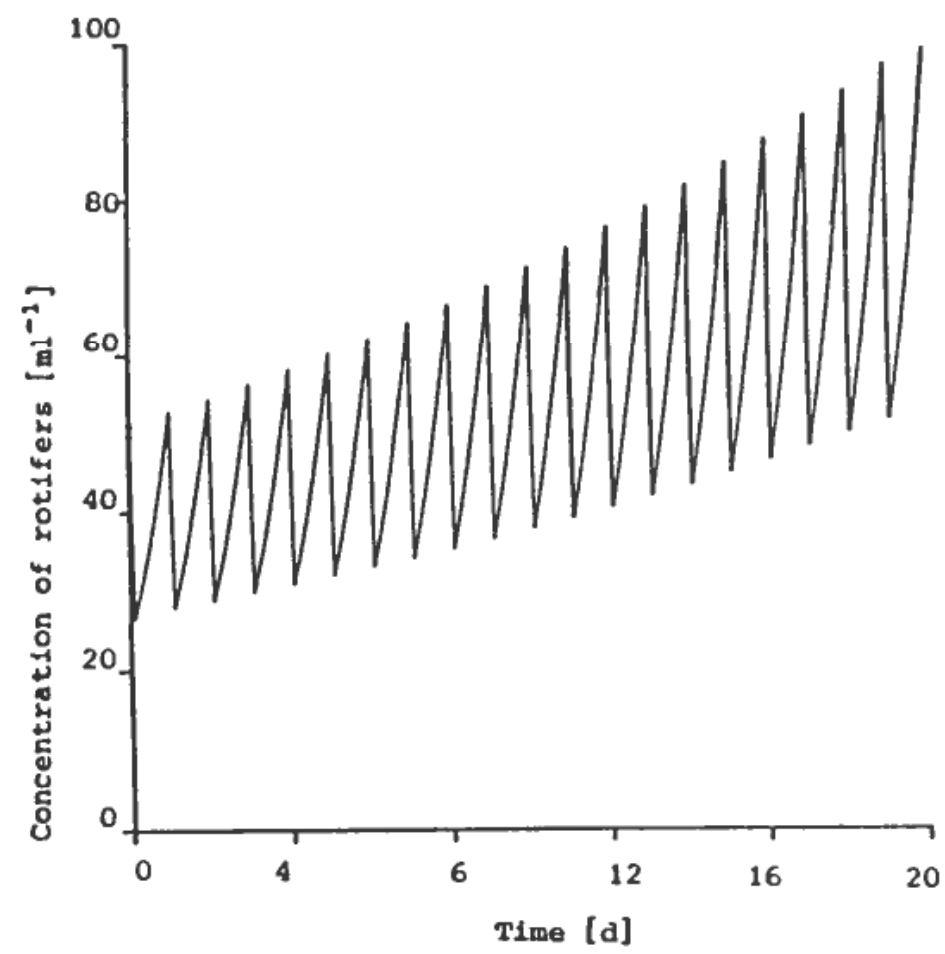

Figure 3. Simulated concentration of rotifers as a function of time after an increase of $10 \%$ in the reproductive rate and using a constant harvest fraction and sufficient supply of food.

When a controller as described in a previous section is used, the harvest fraction will be adjusted to give a steady state level in the rotifers' concentration (Fig. 4). An increase of $10 \%$ in the egg production gives an increase in doubling rate of $5 \%$ and volume fraction necessary to harvest in order to keep a steady state concentration of rotifers by $3 \cdot 3 \%$.

The amount of rotifers that can be harvested depends on the sampling time. In Fig. 5 we have plotted the daily possible yield $\left(\mathrm{cal}^{-1}\right)$ as a function of sampling time, and the results reveal increased yield as the sampling time decreases.

In a production plant for live feed for fish larvae, the demand for rotifers depends on the age structure of the larvae and utilization of other food sources. Variations in the demand for rotifers can be met by changing sampling rate, growth rate or production volume. Changing sampling rate and growth rate can only meet relatively small variation in the demand for rotifers. The most effective way of changing the production capacity is by manipulation of production volume.

We now assume that the rate of newly hatched fish larvae is given as a sinusoidal function of time.

$$
N_{\text {hatch }}=f_{h m x} \sin \left(\frac{\pi t}{S_{p}}\right)
$$

where

$f_{h m x}$ maximum rate of newly hatched fish larvae

$S_{p}$ spawing period in days

$t$ time in days 

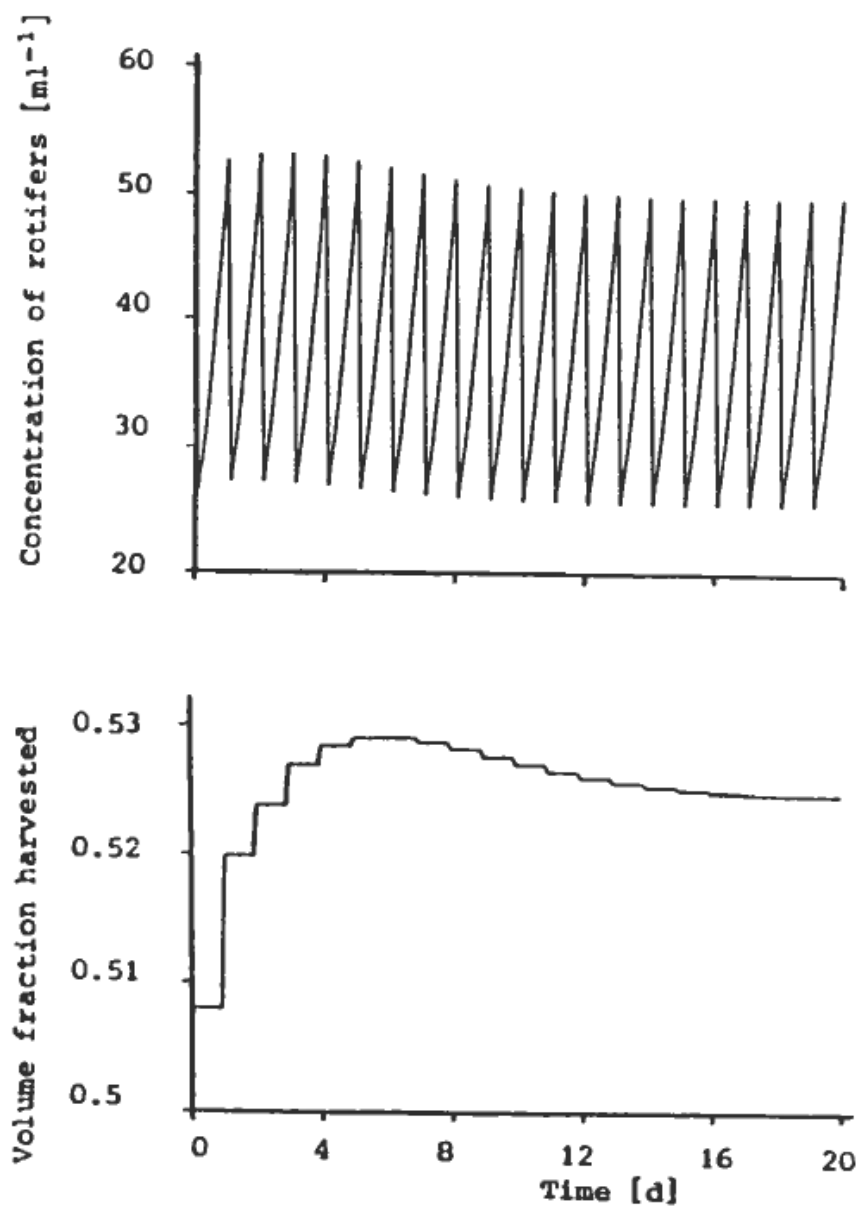

Figure 4. Simulated concentration of rotifers $(a)$ and the necessary volume fraction to harvest each day $(b)$ after an increase in the reproduction rate by $10 \%$.



Figure 5. Daily yield of biomass from one litre of rotifer culture as a function of the sampling time. 


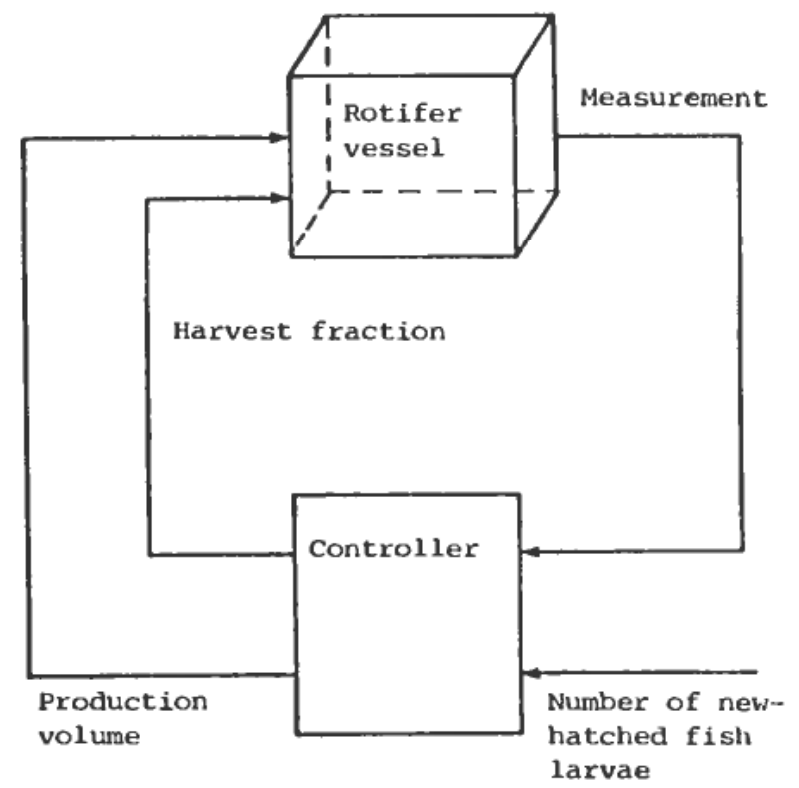

Figure 6. Control strategy for production of rotifers.

The concentration of fish larvae is assumed constant which mean that the culture volume of fish larvae is proportional to the total number of fish larvae. As new volume is added, rotifers are supplied in order to keep the concentration constant.

The decrease in rotifers' concentration due to feeding must also be compensated for. The controller gets information about the number of newly hatched larvae each day and the concentration of rotifers in the rotifer production vessel at each sampling, see Fig. 6.

The amount of rotifers at the next sampling is calculated from the expected larval demand and increase in volume due to supply of newly hatched fish larvae. Using the model, it is simple to calculate necessary start concentration at the present sampling which ensure future needs. In Fig. 7 the necessary production

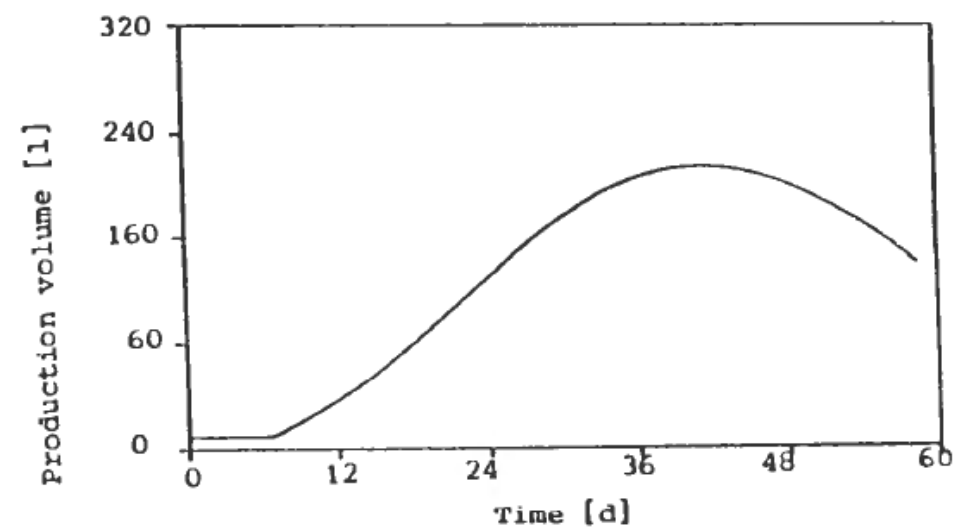

Figure 7. Necessary production volume of rotifers when the rate of newly hatched fish larvae is given as a sinusoidal function of time with a maximum of 1000 larvae $d^{-1}$ at day 30 of the simulation. We observe from the Fig. that the maximum production of rotifers is 2 weeks after the maximum input of newly hatched fish larvae. 


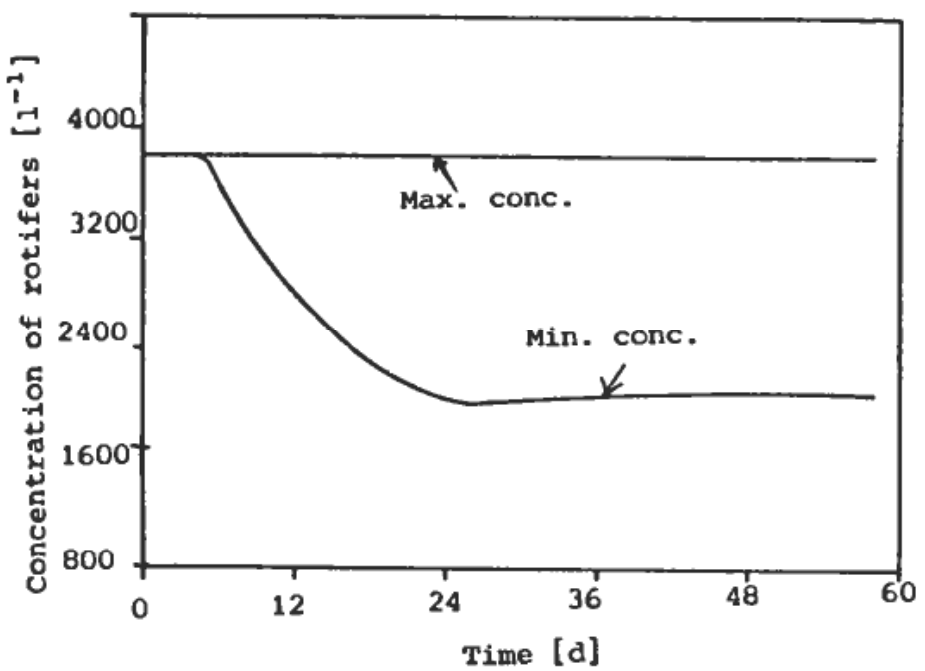

Figure 8. Minimum and maximum concentration of rotifers that the fish larvae see as a function of time. Sampling time is 24 hours.
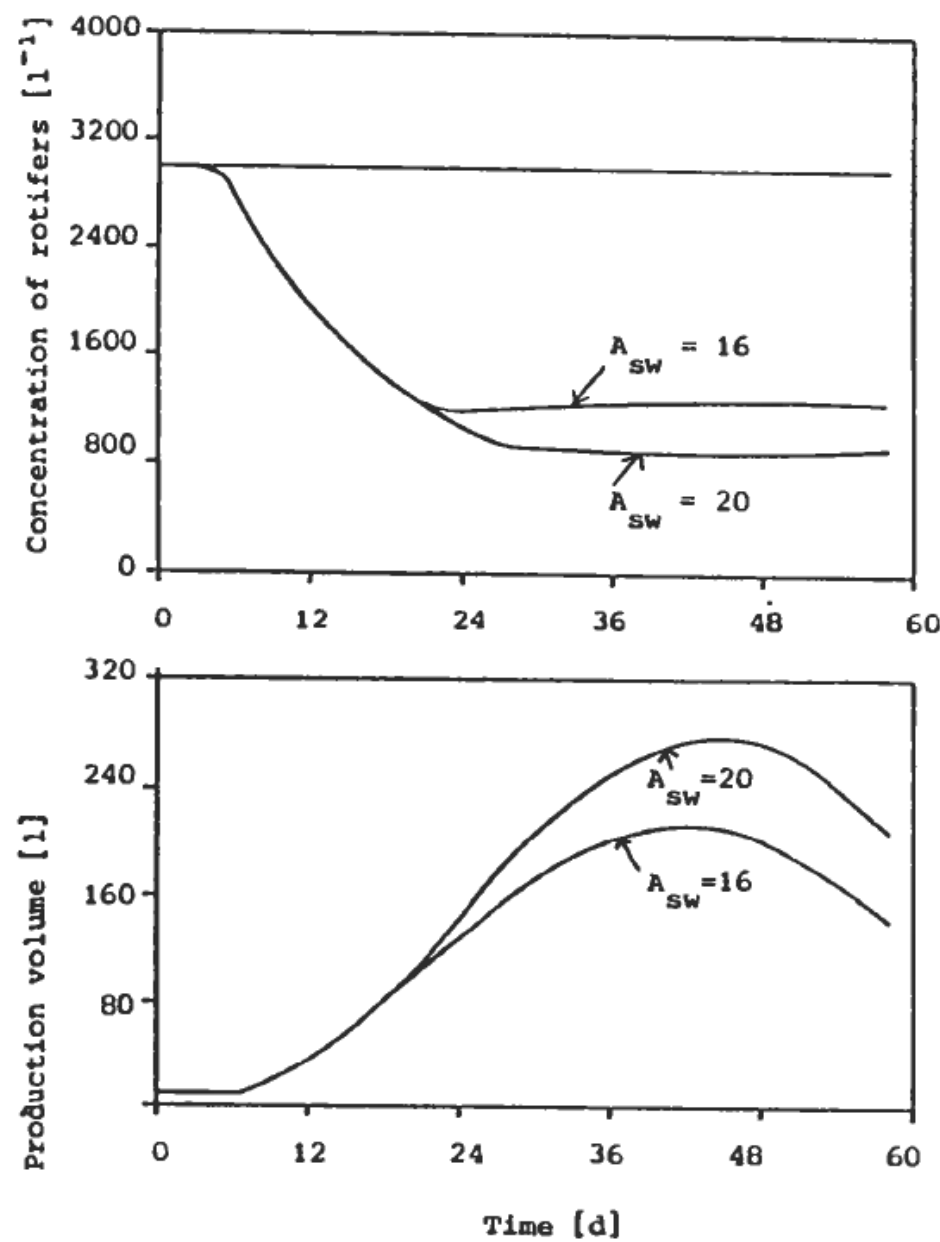

Figure 9. Effect of increasing larval age when switching from rotifers to another food source, $A_{s w}$. (a) Minimum and maximum concentration of rotifers and $(b)$ necessary production volume of rotifers as a function of time. 
volume is plotted as a function of time. The rate of hatching is given by eqn. (5) and the parameters used are found in Table 1.

The concentration of rotifers that the fish larvae see is shown in Fig. 8. The concentration has a variation range of 1800 per litre. If this variation cannot be tolerated, the sampling interval must be shorter or the concentration of fish larvae must be reduced.

The simulation results presented up to now are based on the parameters given in Table 1. These parameters are only tentative and it would be interesting to study the consequences of how change or drift in the parameters would affect the simulation results. The parameter, $A_{s w}$, is the age when the fish larvae start switching from rotifers to another food source. If this switching starts at a higher age, the demand for rotifers will increase. In Fig. 9 the simulation results are shown when the age of switching is increased from day 16 to day 20 . We observe that the necessary production volume is increased to about 30 percent. The variation range in the rotifer concentration of the fish larvae vessel is increased from 1800 to 2100 per litre or 17 per cent.

\section{REFERENCES}

EHrLich, K. F. (1974). Chemical changes during growth and starvation of larval Pleuronectes platessa. Mar. Biol., 24, 39-48.

Mitchell, E., and GAUThIER, J. (1981). Advanced continuous simulation language (ACSL). User Guide/Reference Manual. Mitchell and Gauthier, Assoc., Inc. P.O. Box 685 Concord, Mass. 01742, USA.

SlaGSTAD, D. (1981). Modeling and simulation of physiology and population dynamics of copepods. Effect of physical and biological parameters. Modeling, Identification and Control, 2, 119-162.

Theilacker, G. H., and McMaster, M. F. (1971). Mass culture of the rotifer Brachionus pilcatilis and its evaluation as food for larval anchovies. Mar. Biol., 10, 183-188. 